\title{
Mapping of the hydrophobic composition of lignosulfonates
}

\section{Supporting Information}

Oliver Musl, ${ }^{1}$ Irina Sulaeva, ${ }^{2}$ Ivan Sumerskii, ${ }^{2}$ Arnulf Kai Mahler, ${ }^{3}$ Thomas Rosenau, ${ }^{1}$ Jana Falkenhagen, ${ }^{4 *}$ and Antje Potthast ${ }^{1^{*}}$

${ }^{1}$ Department of Chemistry, Institute of Chemistry of Renewable Resources, University of Natural Resources and Life Sciences, Konrad-Lorenz-Strasse 24, A-3430 Tulln, Austria

${ }^{2}$ Core Facility „Analysis of Lignocellulose” ALICE, University of Natural Resources and Life Sciences, Konrad-Lorenz-Strasse 24, A-3430 Tulln, Austria

${ }^{3}$ Sappi Europe, Sappi Papier Holding GmbH, Bruckner Straße 21, A-8101 Gratkorn, Austria

${ }^{4}$ Bundesanstalt für Materialforschung und -prüfung (BAM), Department Materials Chemistry, Division Structure Analysis, Richard-Willstätter-Straße 11, 12489 Berlin, Germany

* Correspondence: antje.potthast@boku.ac.at; Tel.: +43 14765477412 jana.falkenhagen@bam.de; Tel.: +49 3081041632

Number of pages: 18

Number of figures: 6

Number of tables: 2 


\section{Table of contents}

Page S1 Table S1. Elution program for the $1^{\text {st }}$ dimension (HIC).

Page S1 Figure S1. Calibration plot of polystyrene sulfonate (PSS) standards in aqueous high-speed SEC.

Page S2 Table S2. Calculated statistical moments for lignosulfonate samples based on SEC-MALS.

Page S2 Figure S2. Hemicellulose composition of ultrafiltrated NSSC lignosulfonates.

Page S3-8 Figure S3. HSQC NMR spectra of the used lignosulfonate samples.

Page S9 Figure S4. Normalized chromatograms of lignosulfonate samples in $1^{\text {st }}$ dimension HIC.

Page S10 Figure S5. Relative peak areas of the studied lignosulfonates in HIC (based on UV detection in $1^{\text {st }}$ dimension) plotted against EtOH content in the mobile phase.

Page S11-17 Figure S6. 2D plots from online coupling of HIC and aqueous SEC (big picture size). 
Table S1. Elution program for the $1^{\text {st }}$ dimension (HIC).

\begin{tabular}{ccccc}
\hline Time (min) & Flow rate (mL/min) & Eluent A (\%) & Eluent B (\%) & Eluent C (\%) \\
\hline $\mathbf{0 - 2 0}$ & 0.5 & 100 & & \\
$\mathbf{2 0 - 3 0}$ & 0.5 & 90 & 10 & \\
$\mathbf{3 0 - 4 8}$ & 0.5 & 60 & 40 & \\
$\mathbf{4 8 - 7 6}$ & 0.5 & 15 & 85 & 100 \\
$\mathbf{7 6 - 9 0}$ & 0.5 & & 100 & \\
$\mathbf{9 0 - 1 0 0}$ & 0.5 & & & \\
\hline
\end{tabular}

Figure S1. Calibration plot of polystyrene sulfonate (PSS) standards in aqueous high-speed SEC.

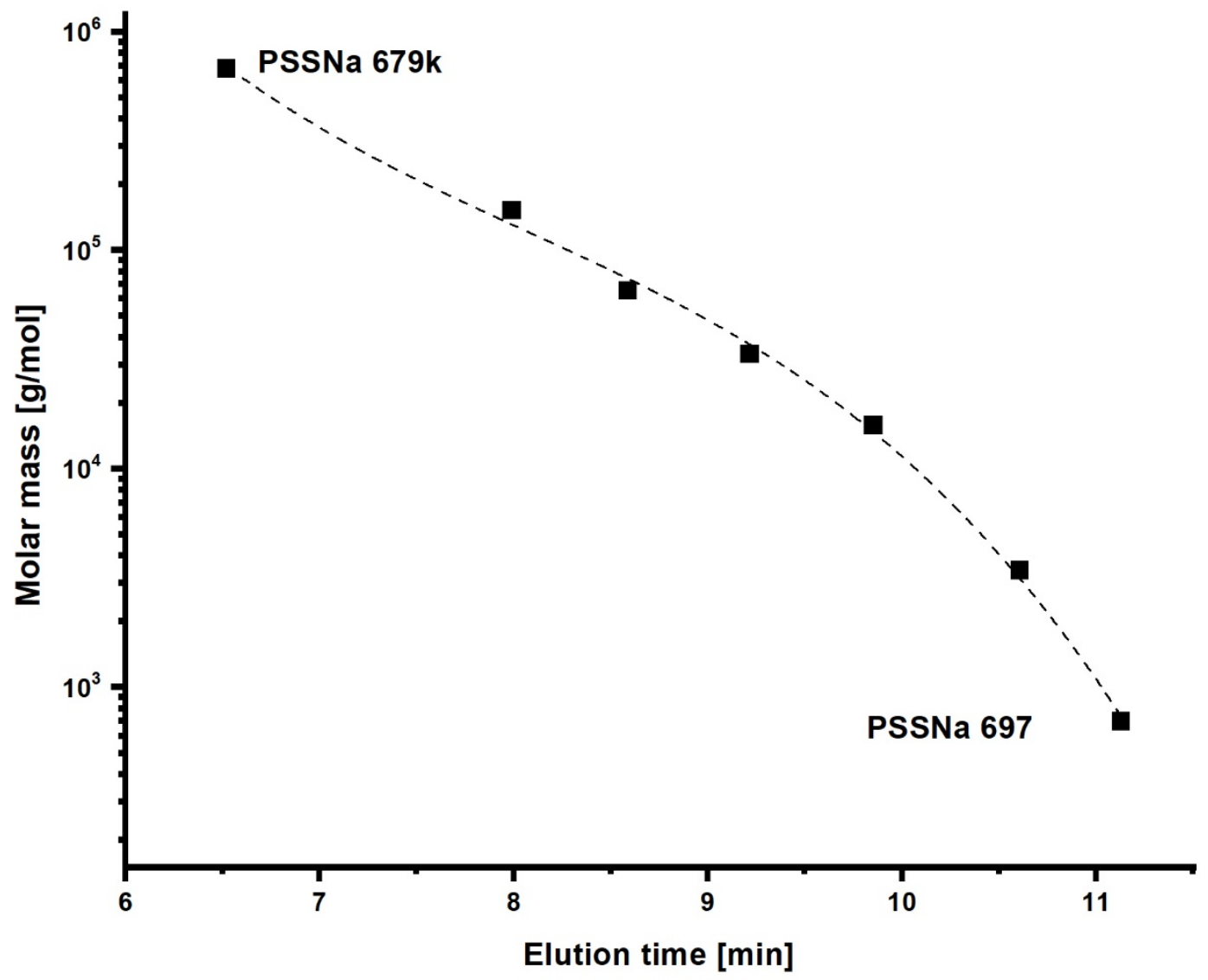


Table S2. Calculated statistical moments for lignosulfonate samples based on SEC-MALS.

\begin{tabular}{|c|c|c|c|c|c|c|}
\hline \multirow{3}{*}{ Nr. } & \multirow{3}{*}{ Sample } & \multicolumn{4}{|c|}{ Statistical moments } & \multirow{3}{*}{$\begin{array}{r}\text { Đ } \\
(\mathrm{Mw} / \mathrm{Mn}) \\
\end{array}$} \\
\hline & & $\mathbf{M}_{\mathbf{n}}$ & $\mathbf{M}_{\mathbf{p}}$ & $\mathbf{M}_{\mathbf{w}}$ & $\mathbf{M}_{\mathbf{z}}$ & \\
\hline & & Da & & & & \\
\hline 1 & NSSCEu\#1 & 1510 & 1600 & 3670 & 9680 & 2.4 \\
\hline 2 & NSSCBe\#2 & 1820 & 9210 & 8650 & 25260 & 4.8 \\
\hline 3 & HWMg\#3 & 2020 & 6210 & 12930 & 52190 & 6.4 \\
\hline 4 & HWNa\#4 & 3150 & 4150 & 25140 & 160670 & 8.0 \\
\hline 5 & SWMg\#5 & 3500 & 19590 & 39720 & 175620 & 11.4 \\
\hline 6 & $\mathrm{SWMgO}_{2} \# 6$ & 9610 & 30130 & 64380 & 247030 & 6.7 \\
\hline 7 & SWMg\#7 & 6150 & 6670 & 40020 & 219570 & 6.5 \\
\hline 8 & SWNa\#8 & 6230 & 19070 & 44700 & 166150 & 7.2 \\
\hline 9 & SWNa\#9 & 5490 & 23800 & 54400 & 183560 & 9.9 \\
\hline 10 & SWNa\#10 & 8410 & 25910 & 57280 & 188910 & 6.8 \\
\hline 11 & SWCa\#11 & 5850 & 19360 & 50920 & 191040 & 8.7 \\
\hline 12 & $\mathrm{SWNH}_{4} \# 12$ & 4410 & 23970 & 44040 & 150250 & 10.0 \\
\hline
\end{tabular}

Figure S2. Hemicellulose composition of ultrafiltrated NSSC lignosulfonates (NSSCEu\#1 and NSSCBe\#2). Samples contained high amounts of xylans. Detected sugar monomers after acid methanolysis: arabinose (Ara), rhamnose (Rha), fucose (Fuc), xylose (Xyl), galacturonic acid (GalUA), 4-O-methylglucuronic acid (4OMeGlcUA), mannose (Man), galactose (Gal), glucose (Glc), and glucuronic acid (GlcUA).

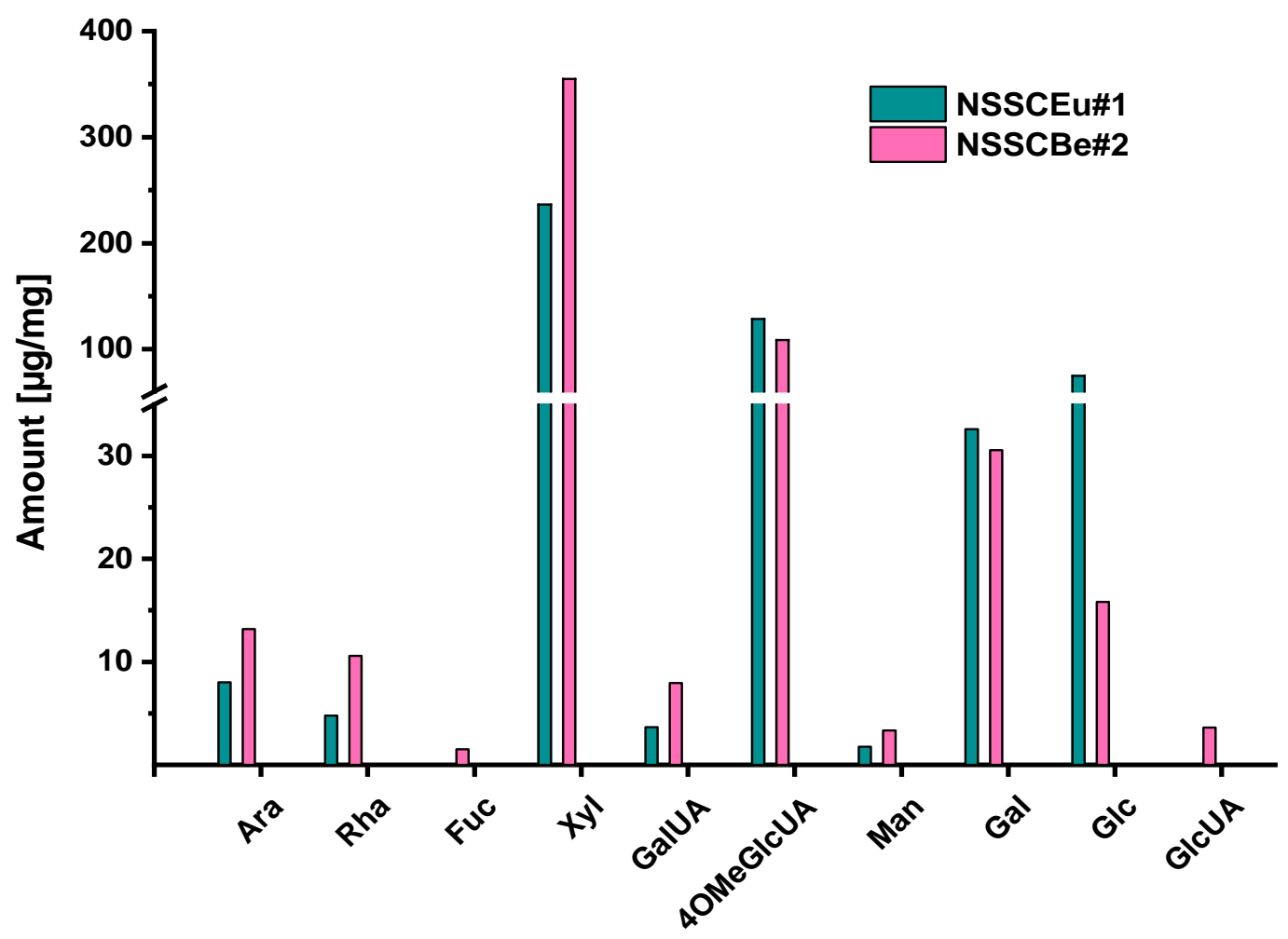


Figure S3. HSQC NMR spectra of the used lignosulfonate samples. Selected structural motifs

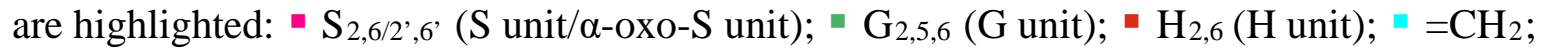

" fa (fatty acids); " $\mathrm{A}_{\alpha, \beta, \gamma}\left(\beta\right.$-aryl ether); - $\mathrm{B}_{\alpha, \beta, \gamma}$ (phenylcoumaran); " $\mathrm{C}_{\alpha, \beta, \gamma}$ (resinol);

- $\mathrm{OCH}_{3}$ (methoxy group); " $\alpha \mathrm{SA}_{\alpha, \beta, \gamma}$ (sulfo group in $\alpha$-position); " xyl (xylans).
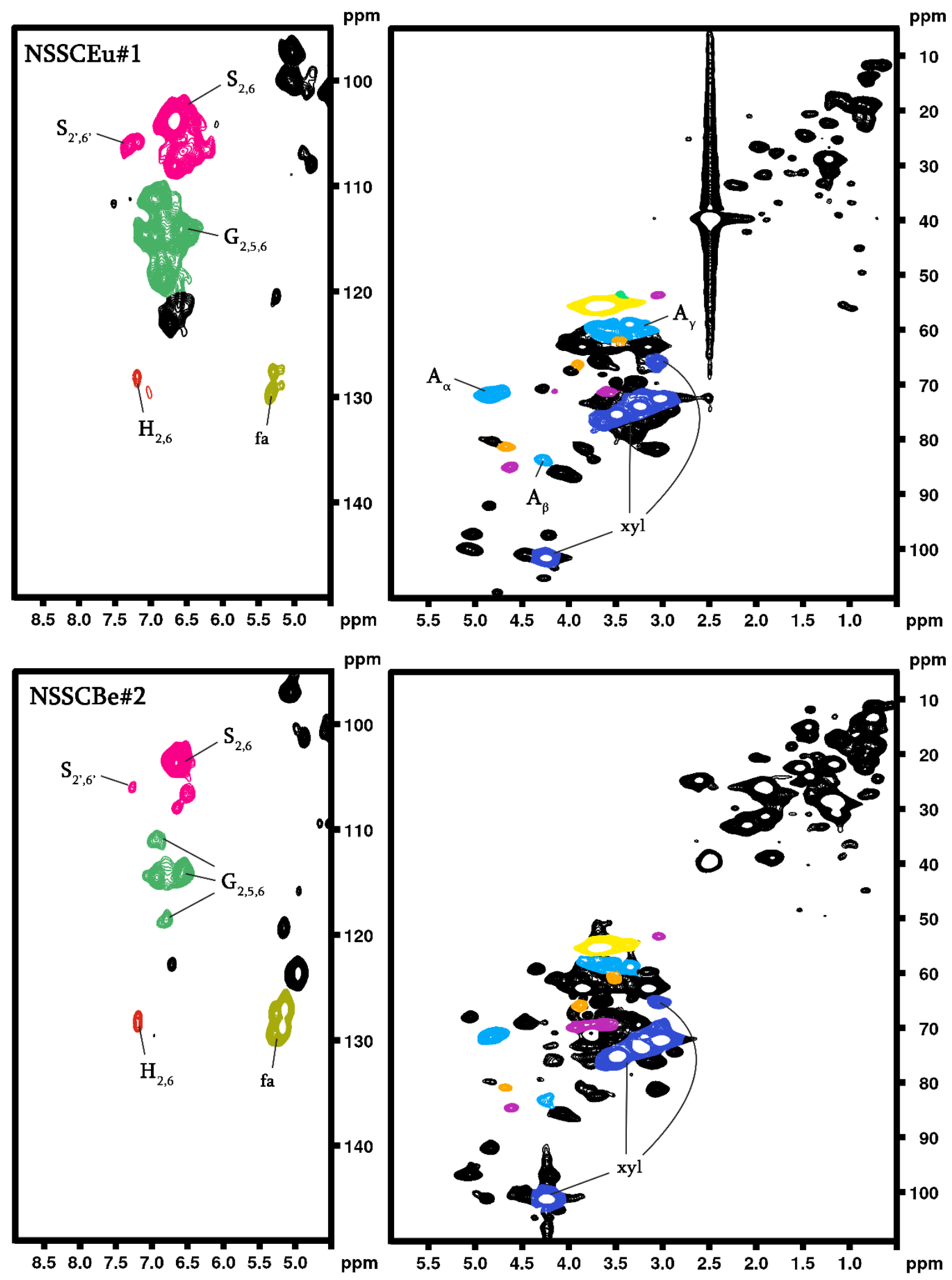


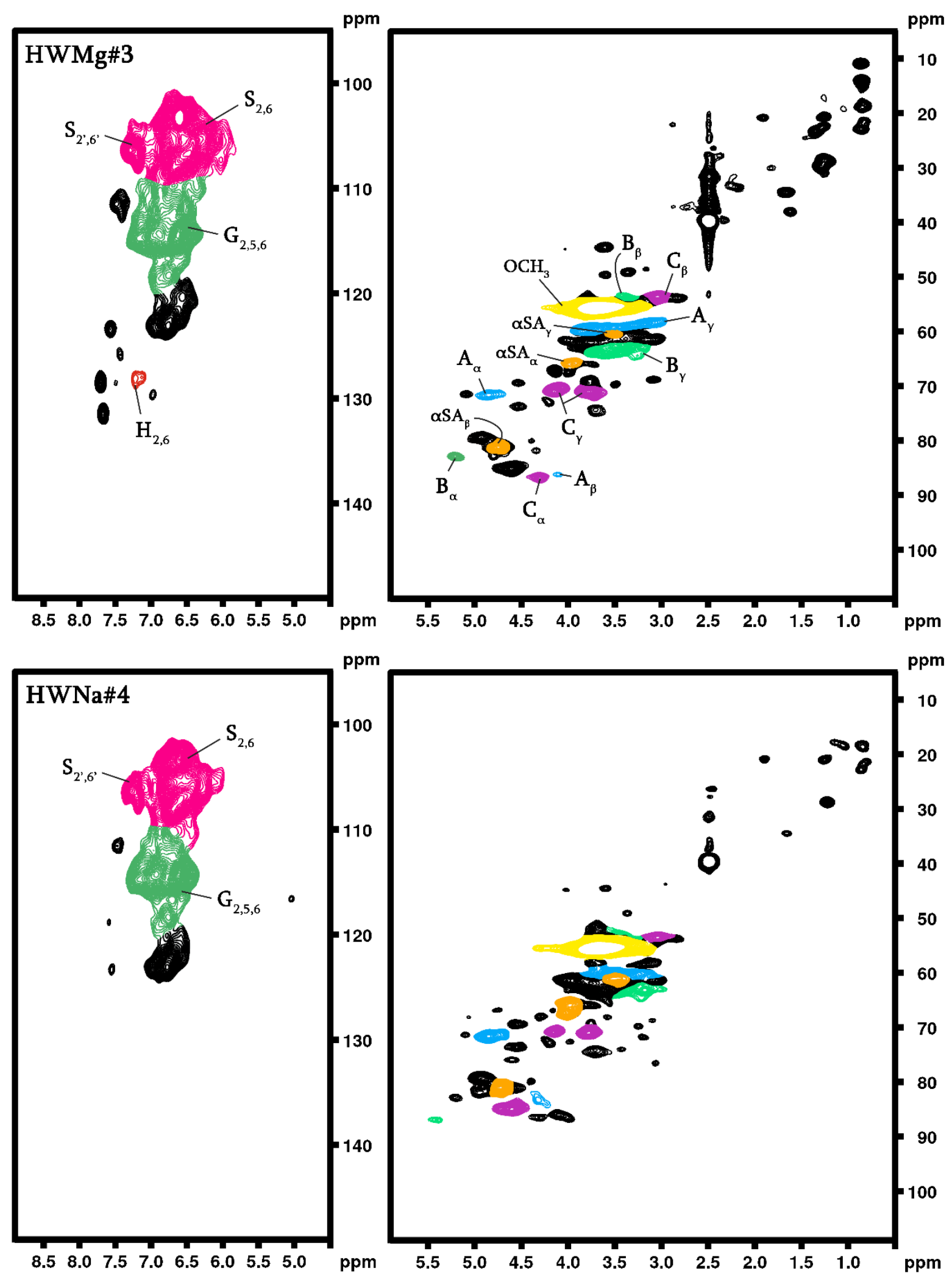




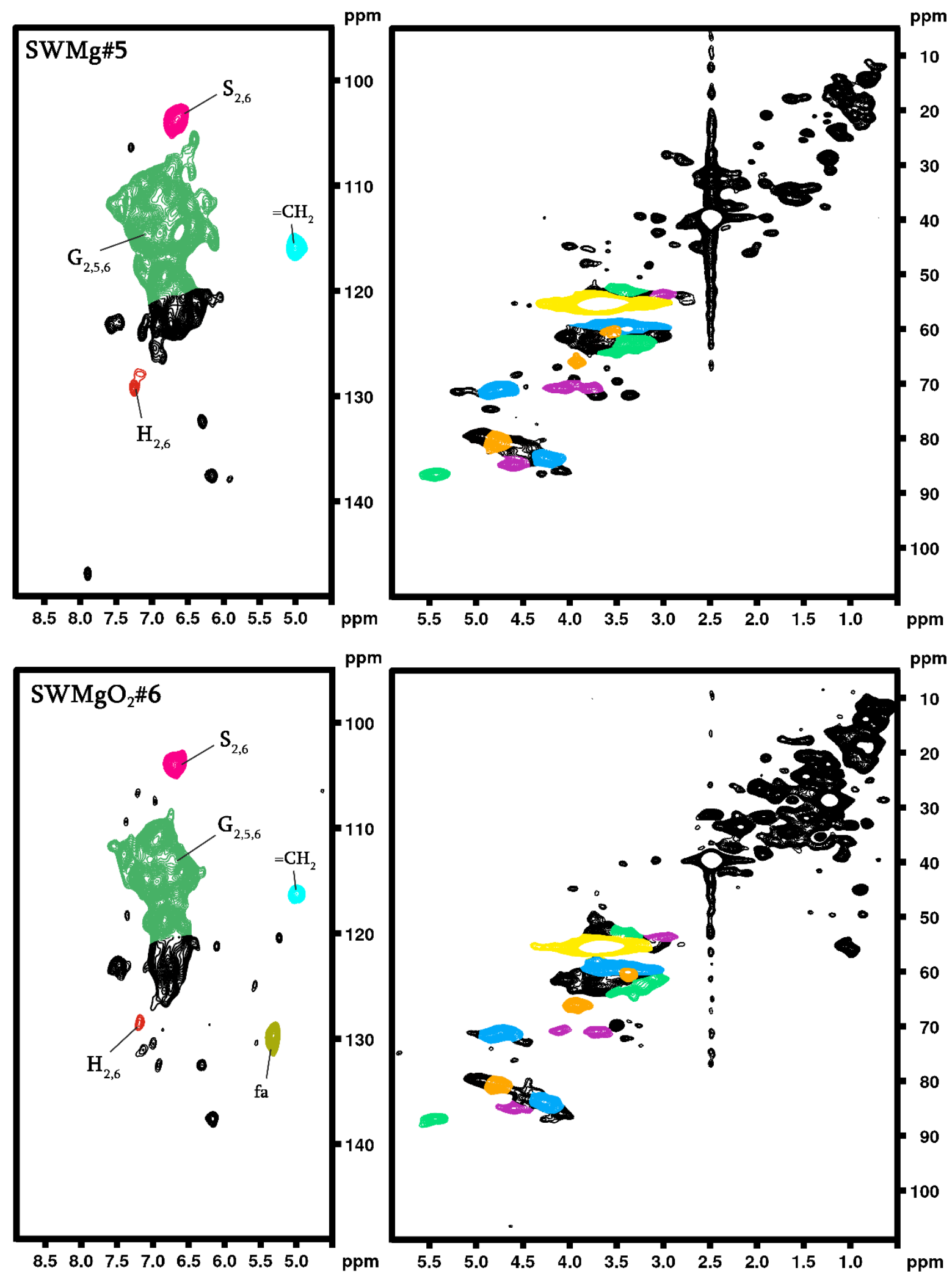




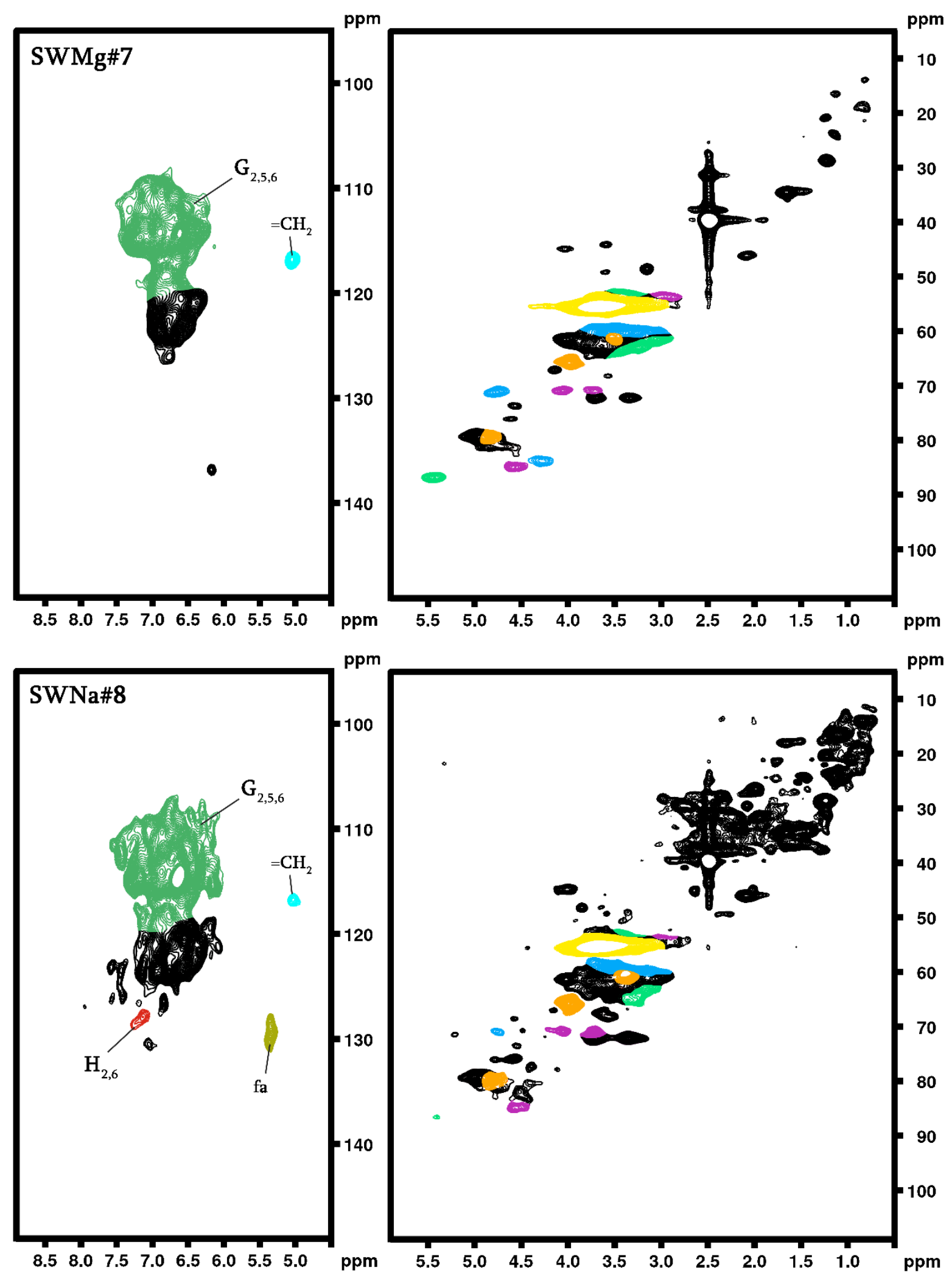




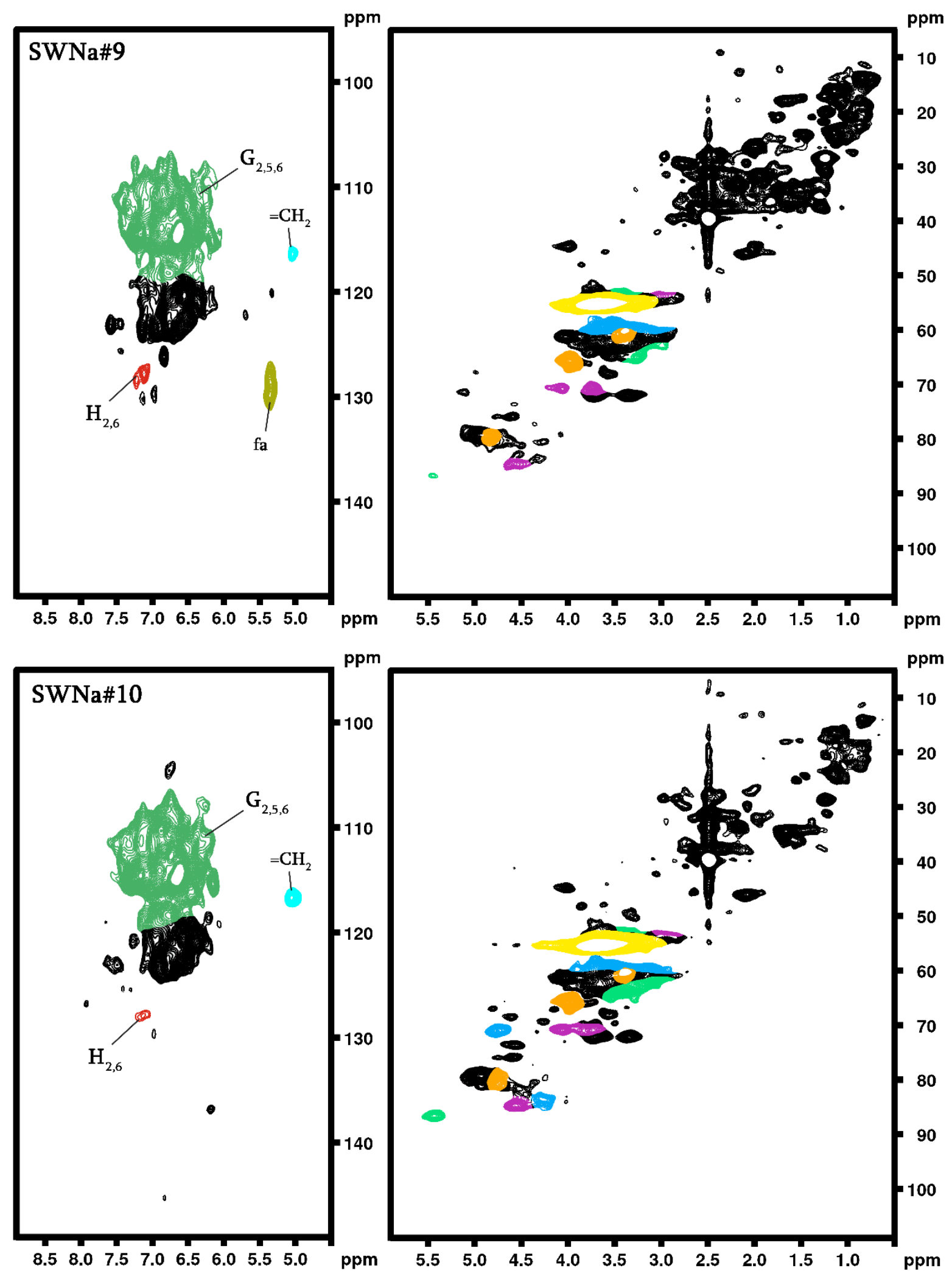




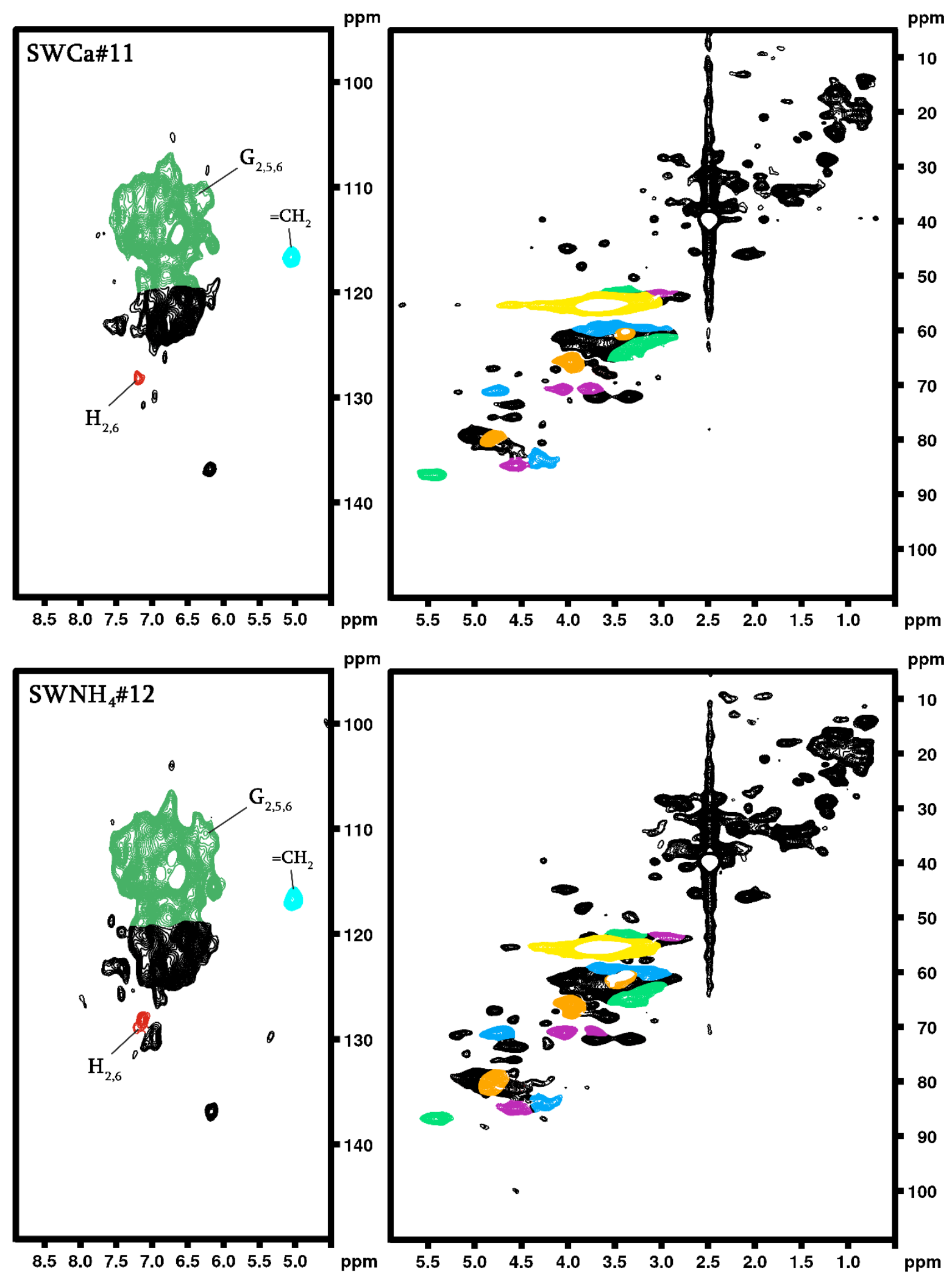


Figure S4. Normalized chromatograms of lignosulfonate samples in $1^{\text {st }}$ dimension HIC. Samples are grouped based on their origin.
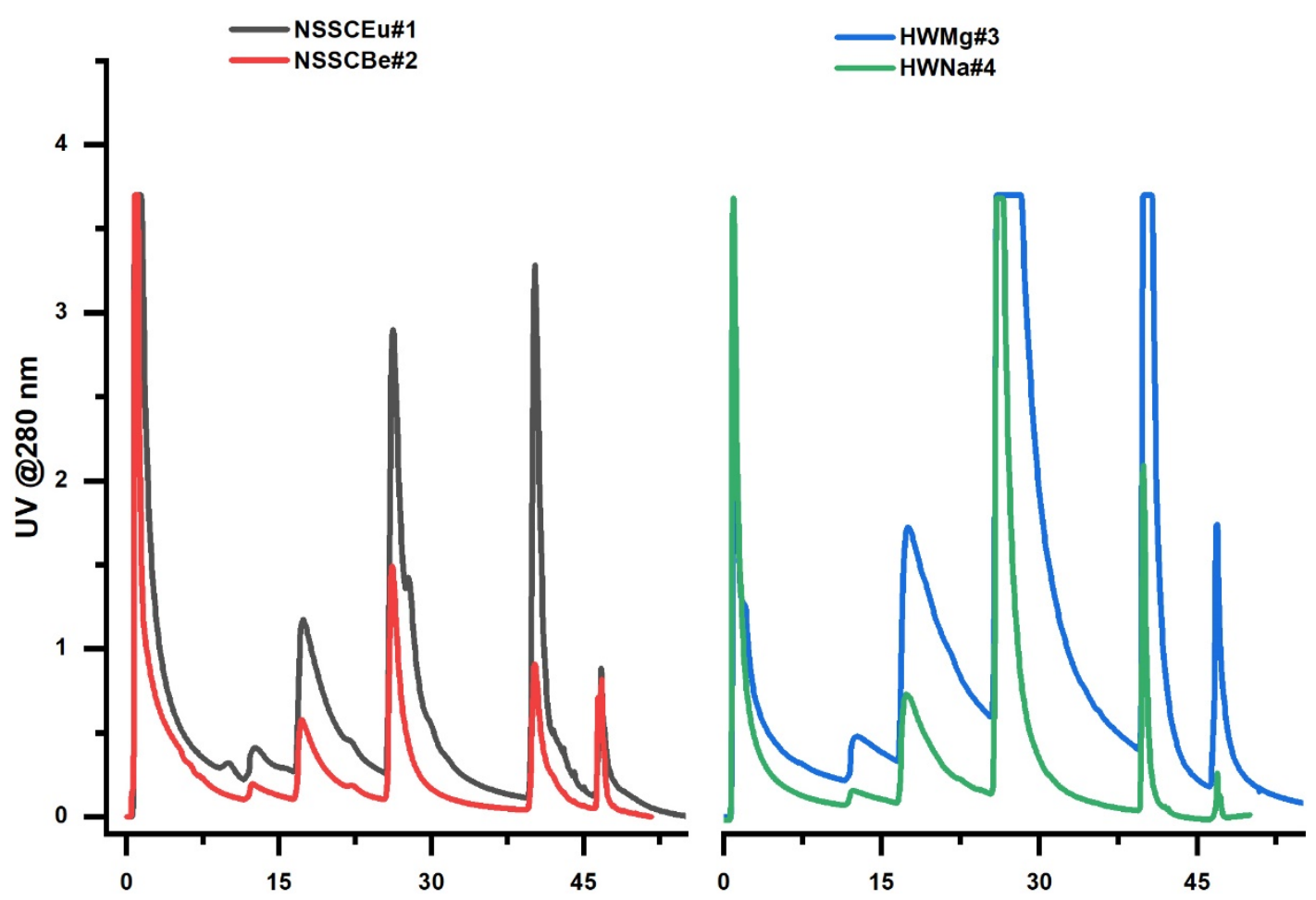

Elution volume $[\mathrm{ml}]$

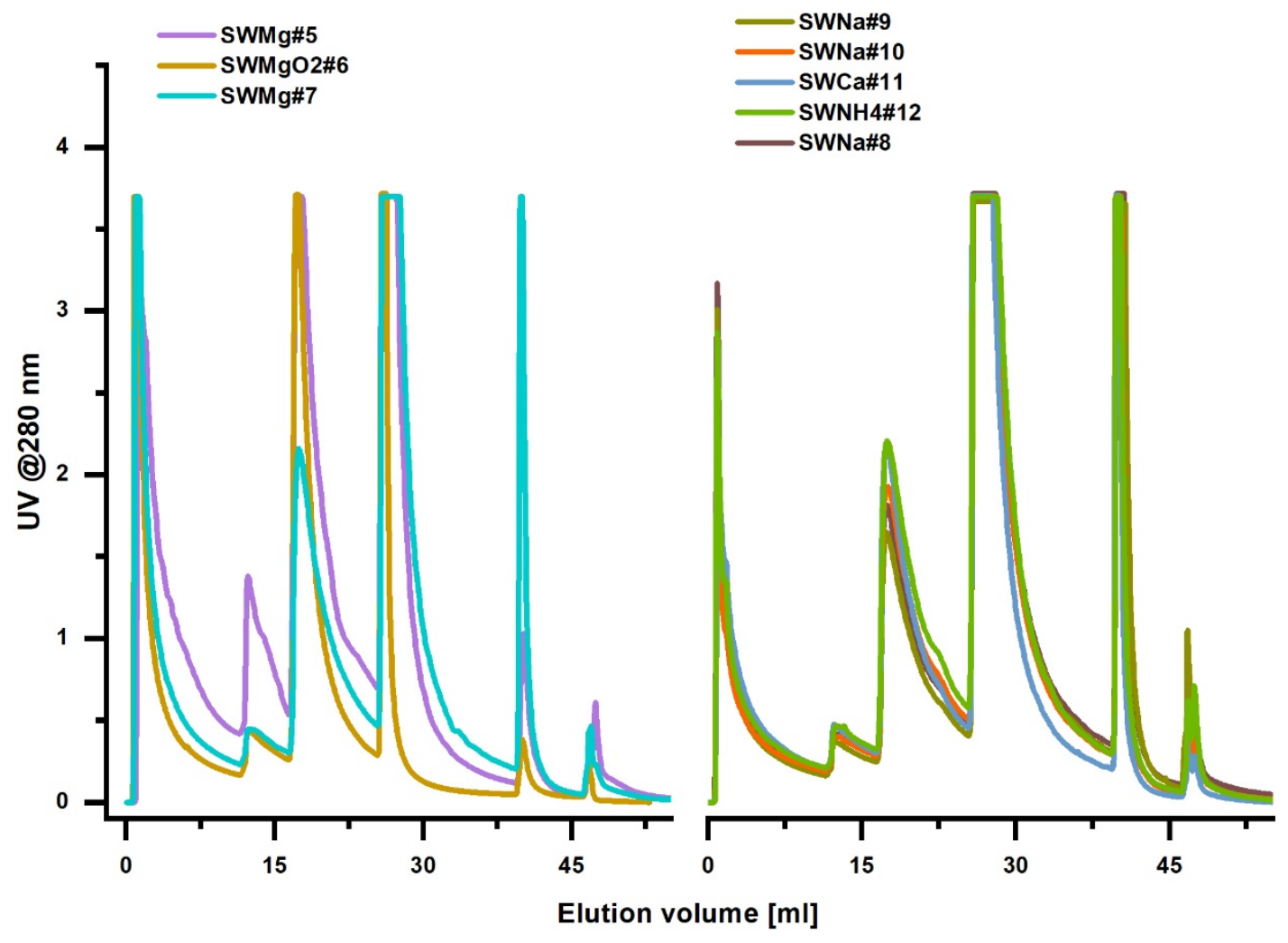


Figure S5. Relative peak areas of the studied lignosulfonates in HIC (based on UV detection in $1^{\text {st }}$ dimension) plotted against EtOH content in the mobile phase. Detector saturation (see chromatograms above) led to distortions in the elution profiles.

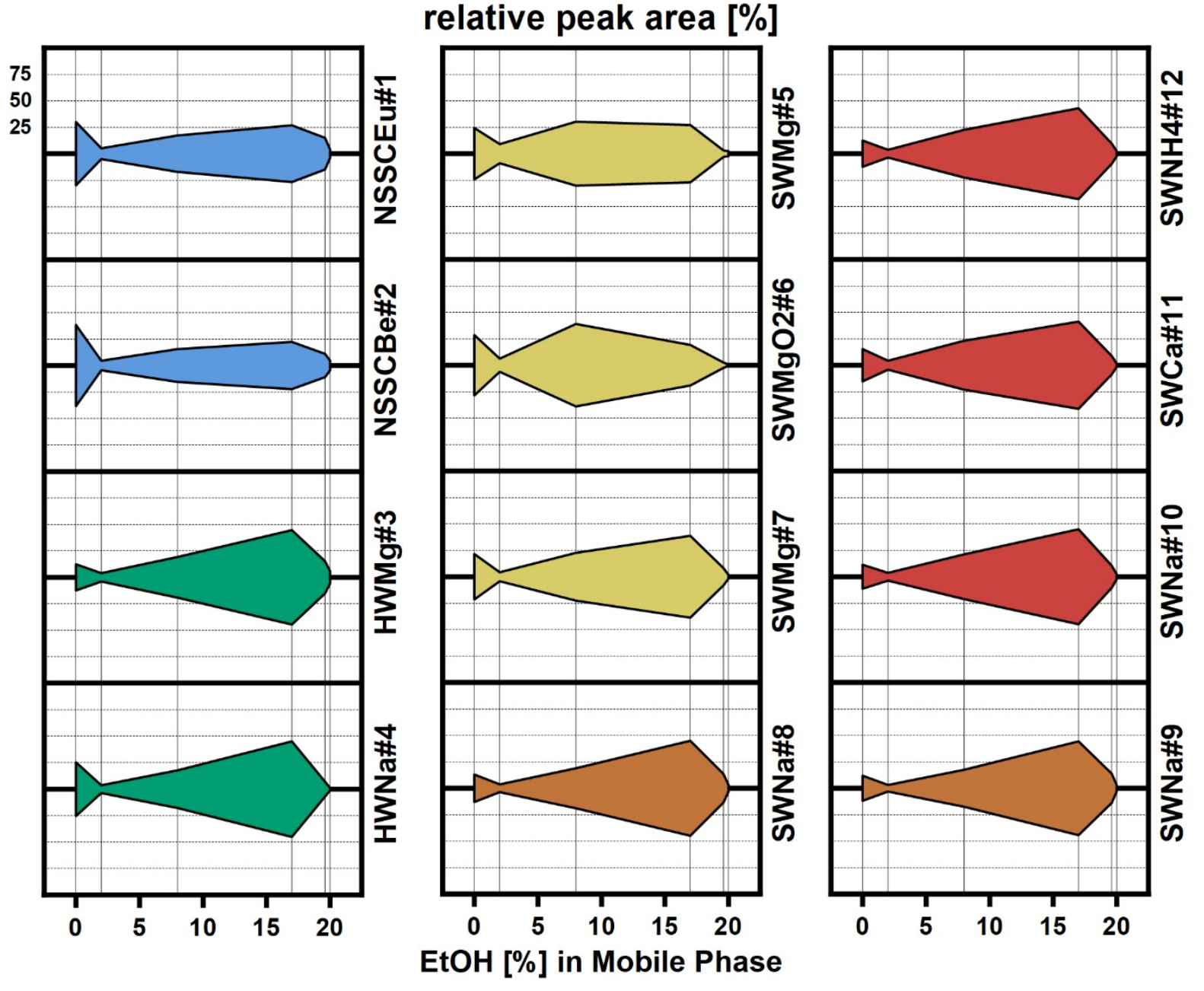


Figure S6. 2D plots from online coupling of HIC and aqueous SEC (big picture size). An increase in retention time in 1st dimension (HIC) is related to an increase in molar mass (shift of peaks towards the upper right corner) and decrease in functionality. Hence, dispersity in chemical composition (CCD) is indicated by a shift towards the center.
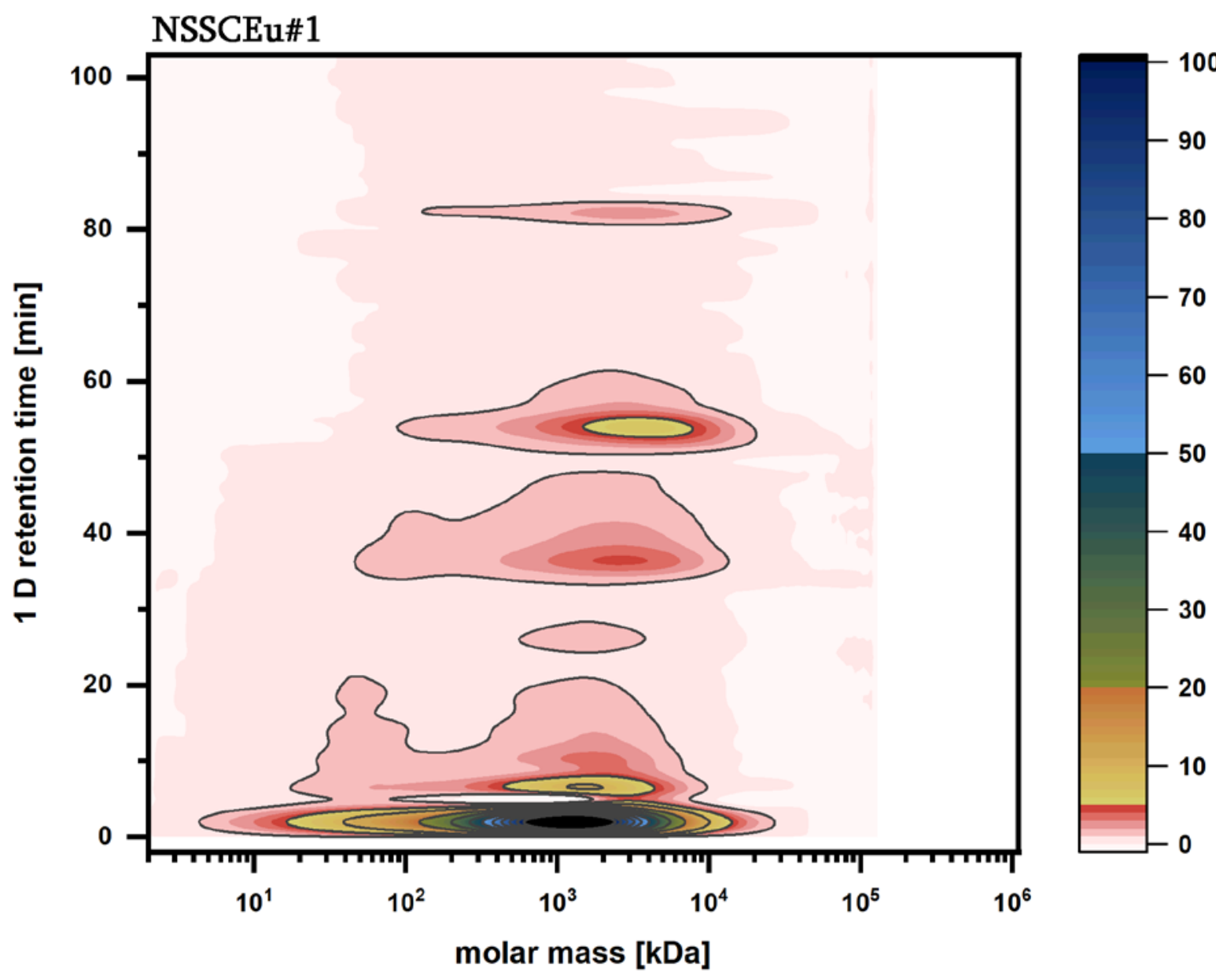

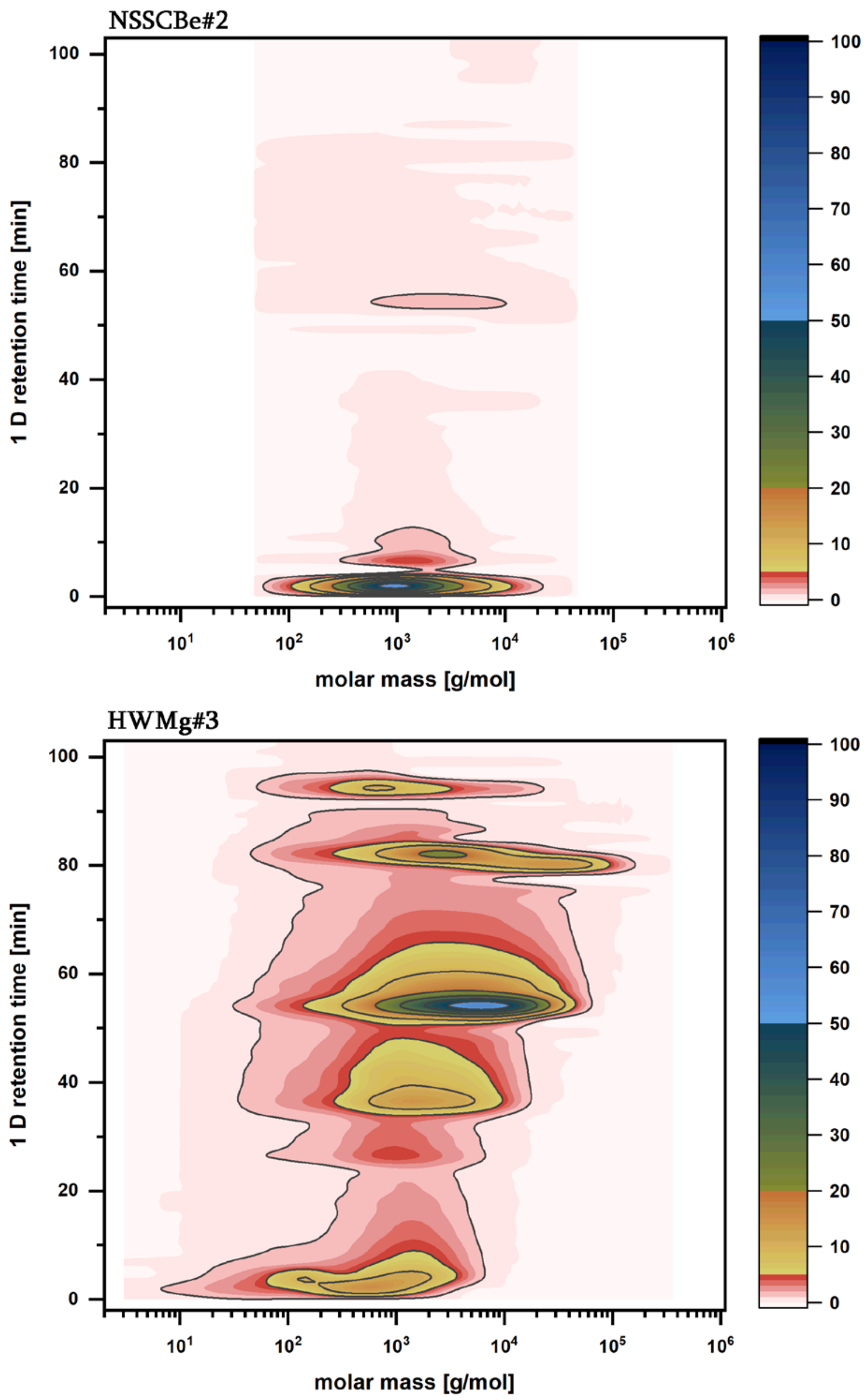

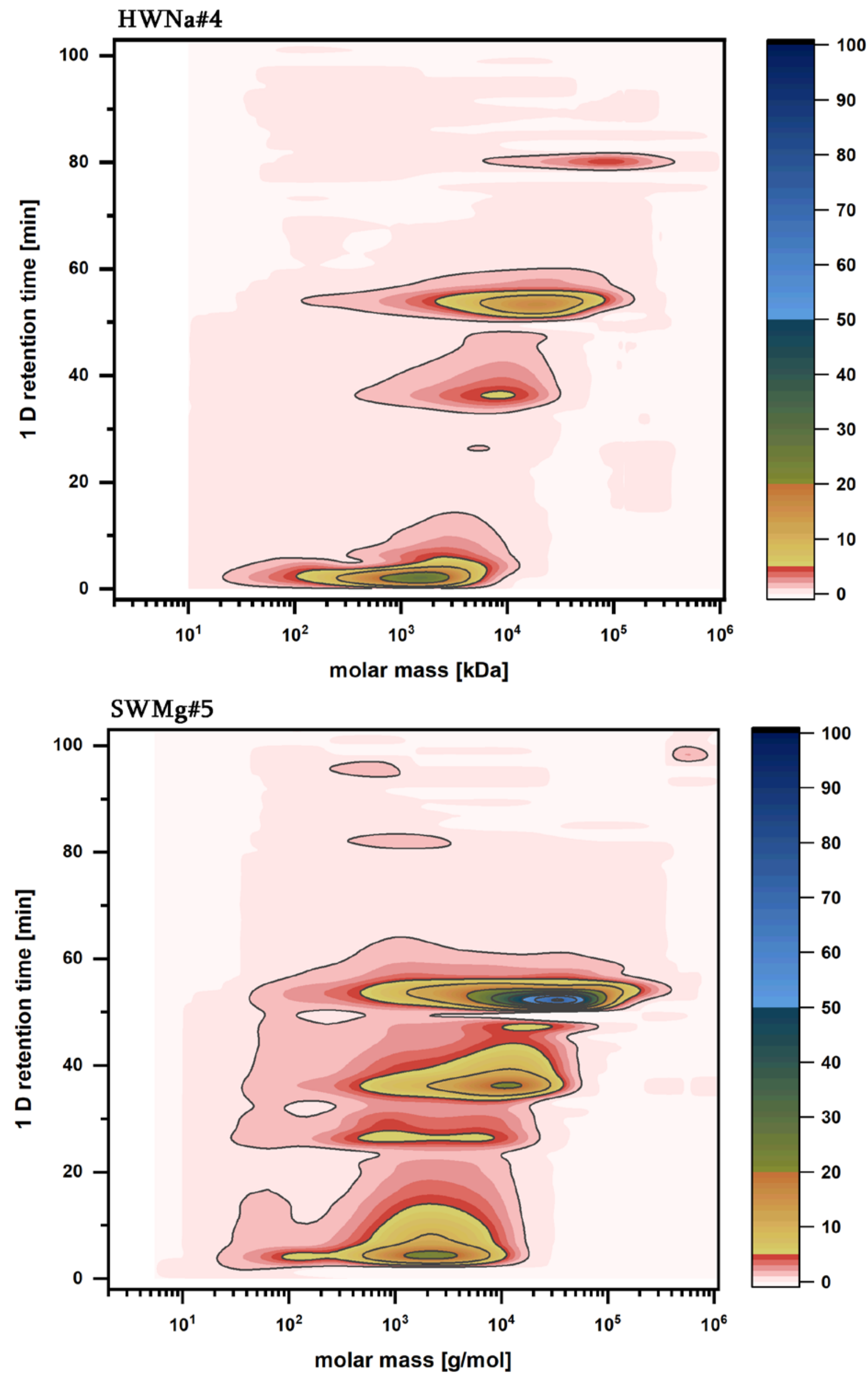

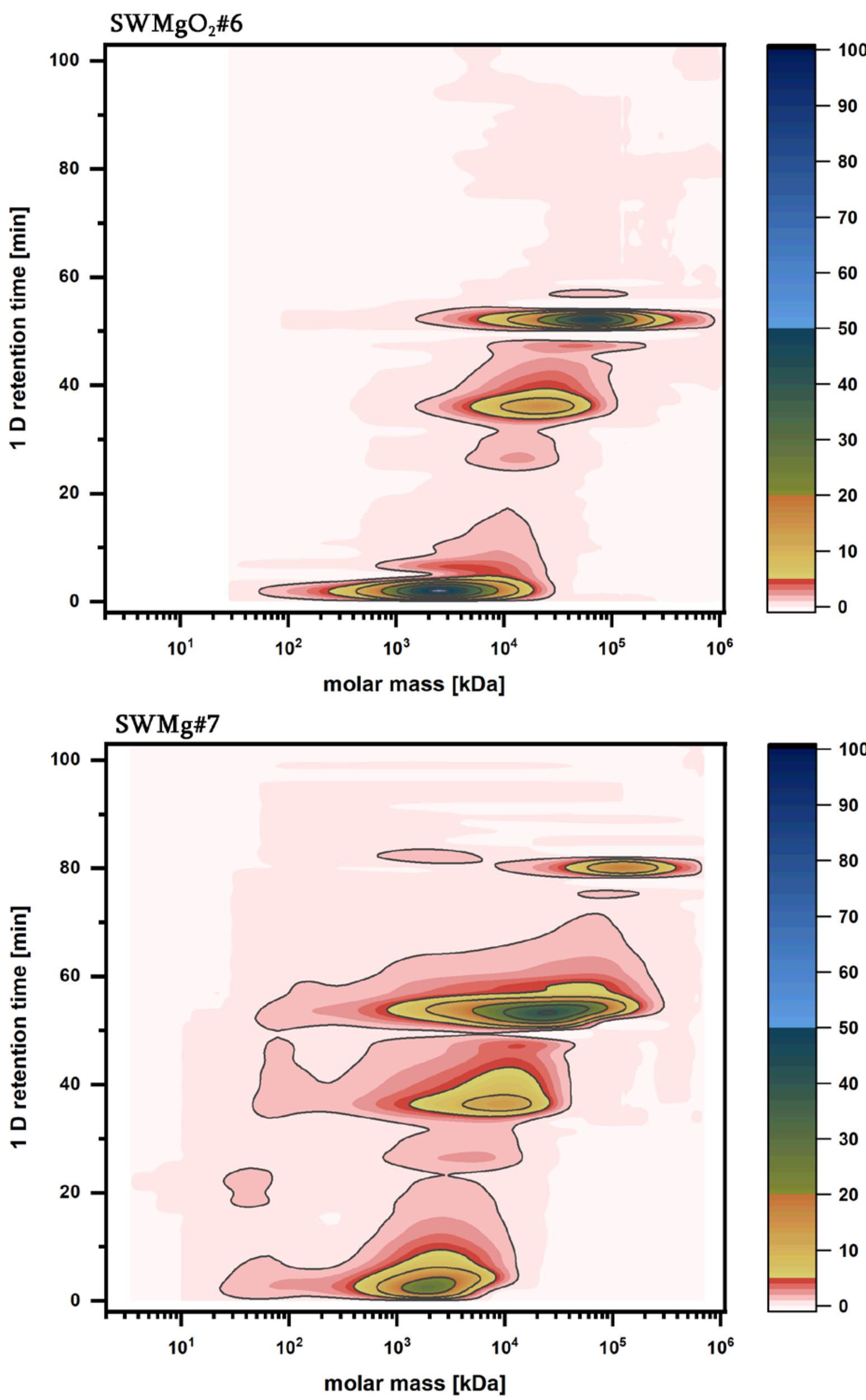

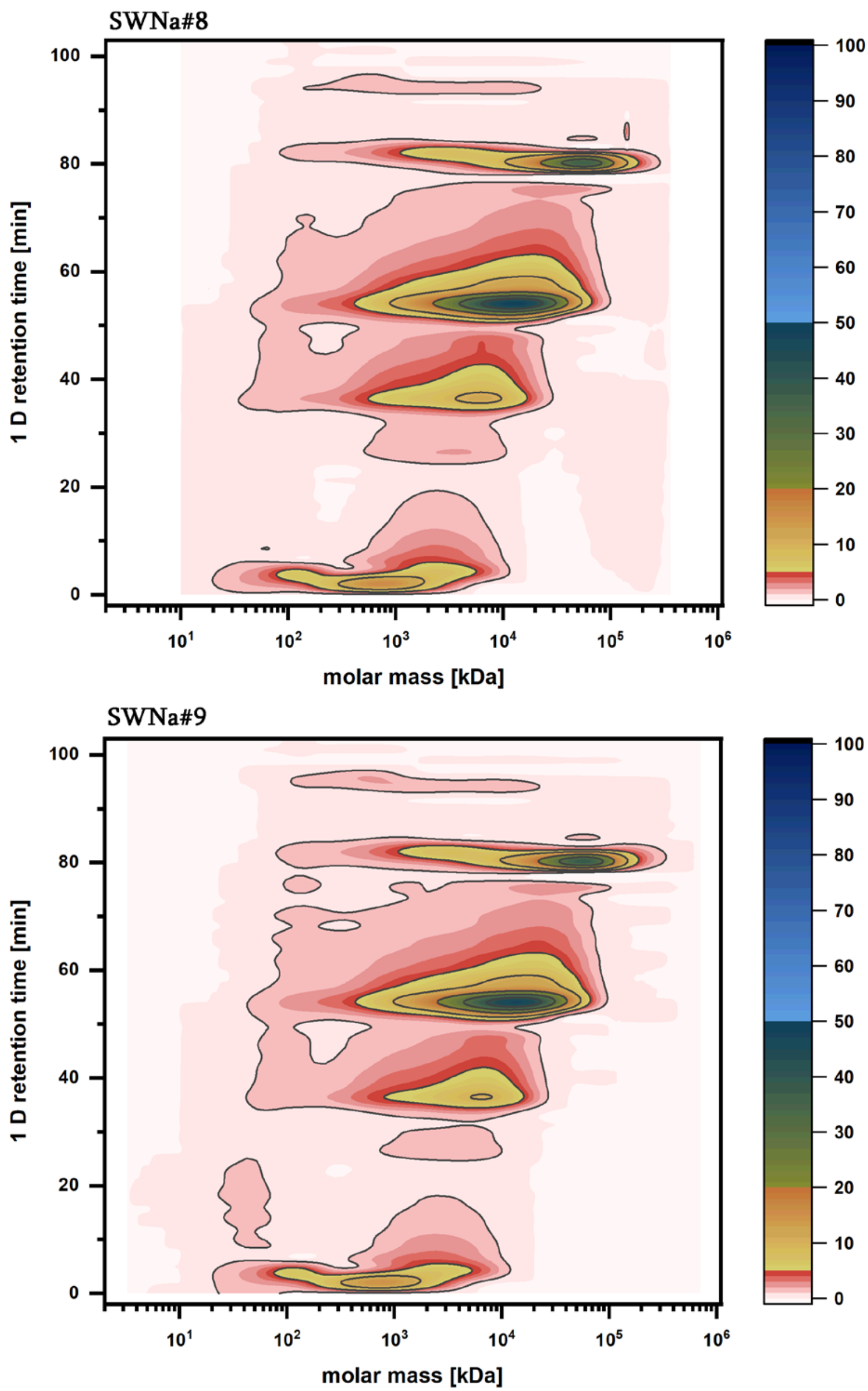

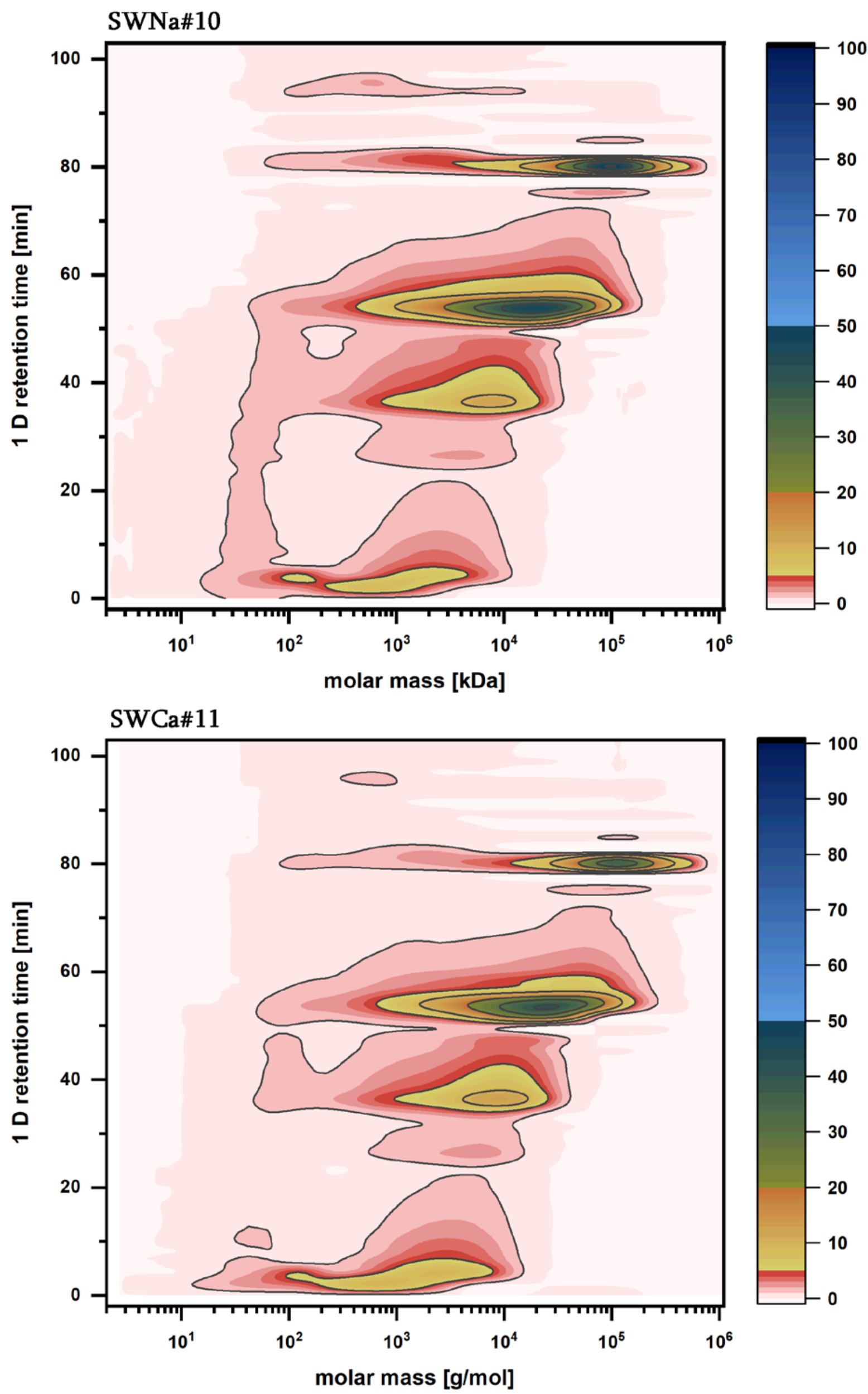


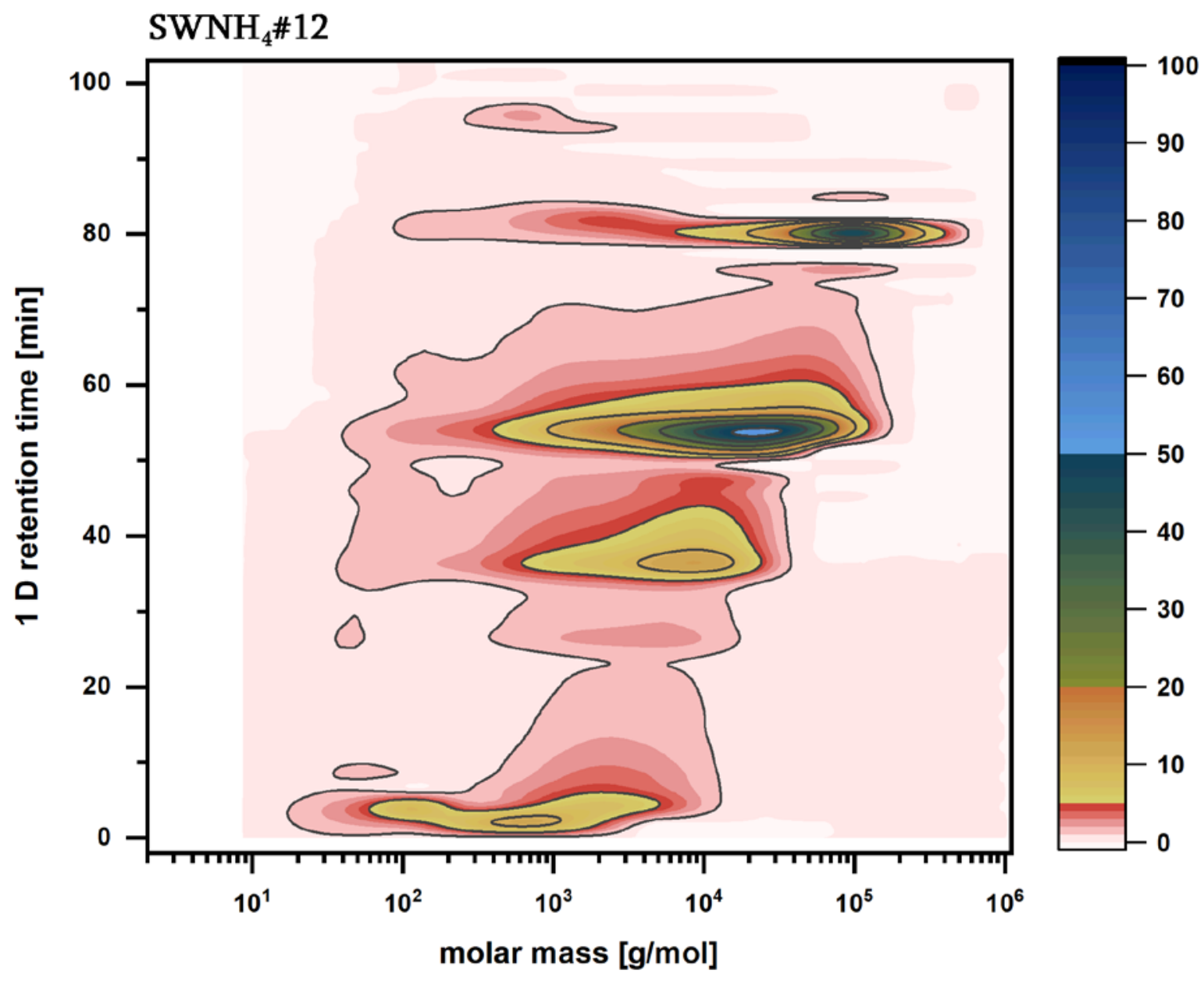

